\title{
Horizontal Low Gradient Magnetophoresis Behaviour of Iron Oxide Nanoclusters at the Different Steps of the Synthesis Route
}

\author{
M. Benelmekki ${ }^{1}$, C. Caparros ${ }^{1}$, A. Montras ${ }^{2}$, R. Gonçalves ${ }^{1}$, \\ S. Lanceros-Mendez ${ }^{1}$ and Ll.M. Martinez ${ }^{3}$ \\ ${ }^{1}$ Centro de Fisica, Universidade do Minho, 4710-057 Braga, Portugal \\ ${ }^{2}$ Sepmag Tecnologies, Parc Tecnologic del Valles, E-08290 Barcelona, Spain \\ ${ }^{3}$ Sepmag Technologies, 191 Peachtree St. NE, 03030 Atlanta. GA, USA. \\ +351253604060 \\ +351253604061 \\ benelmekki@fisica.uminho.pt
}

In this work the use of Horizontal Low Gradient Magnetic Field (HLGMF) $(<100 \mathrm{~T} / \mathrm{m})$ for filtration, control and separation of synthesized magnetic nanoparticles (NPs) is investigated. The characteristics of the suspension, size and type of the NPs are considered and discussed. For these purposes, $\mathrm{Fe}_{2} \mathrm{O}_{3}$ silica coated nanoclusters of about $150 \mathrm{~nm}$ are synthesized by co-precipitation, monodispersion and silica coating. SQUID, TEM, XRD, and $\zeta$ potential techniques were used to characterize the synthesized nanoclusters. An extensive magnetophoresis study was performed at different magnetophoretical conditions. Different reversible aggregation times were observed at different HLGMF, at each step of the synthesis route. In particular, differences of several orders of magnitude were observed when comparing citric acid modified NPs with silica coated nanoclusters . Reversible aggregation times are correlated to the properties of the NPs at different steps of synthesis route.

Keywords: Magnetic nanoparticles, Citric Acid, Reversible aggregation, surface potential, silica coated nanoclusters

\section{Introduction}

In recent years, substantial progress has been made in developing technologies in the field of magnetic microspheres, magnetic nanospheres and ferrofluids. Nanospheres and microspheres containing a magnetic core in a nonmagnetic matrix are used in numerous biological applications (Sayed et al. 2003). They are 
used, for example, as carriers which can be targeted to a particular site by using an external magnetic field.

Magnetic separation of organic compounds, proteins, nucleic acids and other biomolecules and cells from complex reaction mixtures is becoming the most suitable solution for large production in bioindustrial purification and extraction processes. For in-vivo applications, it is important that well-defined biocompatible coatings surround the magnetic particles to prevent any aggregation, and also enable efficient protection of the body from toxicity. However, for In-vitro applications, biocompatible coatings are not indispensable; particles could be coated with non-toxic materials inert to chemical and biological mediums. The current commercial nanoparticles for in vitro application are coated with polystyrene, different copolymers, or silica (Wilson et al. 2002; Tartaj et al. 2003; Wilson et al. 2005).

Due to the low surface/volume ratio of the microspheres, and the unclear magnetic behaviour of the particles, commercial magnetic micro and nano beads have low efficiency for large volume production and potential industrial application. These limitations difficult their use as efficient and low cost carriers for bioindustrial purification and extraction processes (Benjamin 1999).

Scientific bibliography shows the important labours for the improvement and the understanding of the magnetic behaviour of the magnetic nanoparticles and magnetic micro and nano spheres. Most of the scientific studies converge on liquid-phase synthesis of the magnetite particles. Despite their lower magnetic susceptibility, magnetite and maghemite are particularly desirable to synthesis by liquid-phase due to the potential as oxidatively stable nanomagnetic particles with diverse application (Siraprapa et al. 2008). The liquid-phase process of magnetic nanoparticles synthesis is very laborious and comprises different stages, 
normally developed under atmospheric conditions: precipitation, stabilization, coating, and suspension.

The essentials steps in the synthesis and the different applications are the manipulation, recovering, and the removal of the magnetic particles using external magnetic fields (magnetophoresis) (Friedman et al. 2005). In the simplest form, magnetic separation can be achieved by placing a permanent magnet in the proximity of the suspension containing the magnetic particles conjugated with the captured targets. This approach is limited to separate micron scale magnetic beads. More sophisticated magnetic separation devices were developed to capture magnetic particles during continuous flow. These flow-trough devices generally fall into columnar flow devices, planar flow devices, or devices that combine columnar and planar flow systems (Earhart et al. 2009). Columnar flow systems consist on a large flow column ranging from millimeters to tens of centimeters in the size, accompanied with an assembly of large external permanent magnets or electromagnets, witch generate a non uniform field to capture the magnetic particles (Chen et al. 2007). These devices suffer from low field gradients and require conjugates beads to be highly magnetic. Planar flow devices consist on flow channels with dimensions on the order of ten microns. Magnetic material or electromagnets are patterned directly next to or inside the flow channel. These devices offer high magnetic gradients, but the small cross-sectional area and the limited number of flow channels restrict the throughput (Smistrup et al. 2006).

In this work a new approach to the understanding of magnetic NPs and nanoclusters is reported. We synthesised silica coated nanoclusters in three steps, co-precipitation, monodispersion and silica coating. An extensive magnetophoresis study was performed using Horizontal Low Gradient Magnetic Field (HLGMF) (Sepmag Technologies). These systems provide higher magnetic 
forces implying faster separation and lower magnetic nanoparticle losses and the developed processes are easily scalable for industrial application. As reported by De la Cuevas et al. (2008), the magnetophoresis process at HLGMF is driven by a cooperative phenomenon consisting of reversible aggregation of the particles, followed by the moving of the aggregates to the walls of the bottle. Different reversible aggregation times were observed at different HLGMF, at each step of the synthesis route: differences of several orders of magnitude where observed when comparing CA-modified NPs suspension with silica coated nanoclusters suspension. The magnetophoretical behaviour was studied at different $\mathrm{pH}$ of CAmodified NPs. This study improves the understanding on magnetic NPs and nanoclusters for their implementation in different applications.

\section{Materials and methods}

\section{Synthesis of $\mathrm{Fe}_{2} \mathrm{O}_{3}$ silica coated nanoclusters}

Nanoclusters were prepared in three steps. The first step consisted on the coprecipitation of Iron Oxide NPs from an aqueous mixture of FeSO4 and $\mathrm{FeCl} 3$. (1:2 molar ratio) $\mathrm{NH} 4 \mathrm{OH}$ was used as a precipitation agent. For these purpose, FeSO4 $7 \mathrm{H} 2 \mathrm{O}(0.5 \mathrm{mM}, 1.4 \mathrm{~g}$ in $50 \mathrm{~mL})$ and FeCl3.6H2O (1 mM, $2.7 \mathrm{~g}$ in $50 \mathrm{~mL})$ were mixed and heated to $80^{\circ} \mathrm{C}$. In order to precipitate the iron hydroxides, the $\mathrm{pH}$ value was raised and maintained to $\mathrm{pH}=3$ for $30 \mathrm{~min}$. The solution was rigorously stirred at a constant temperature during all the process. Then, the $\mathrm{pH}$ value was increased to 10.5 - 11.

In the second step of the process, Citric Acid (CA) was used to stabilize the magnetic nanoparticles (NPs), according to the method proposed by Campelj et al. (2008). Approximately $2 \mathrm{~g}$ of NPs were mixed with $65 \mathrm{~mL}$ of an aqueous solution of $2 \% \mathrm{CA}(0.02 \mathrm{mg} \cdot \mathrm{mL}-1)$. The $\mathrm{pH}$ value of the mixture was adjusted to $\mathrm{pH}=5$ 
and then it was raised to $\mathrm{pH}=10$ using concentrated ammonia. The adsorption step of the CA was maintained at $80^{\circ} \mathrm{C}$ and rigorously stirred for $60 \mathrm{~min}$. The prepared suspension was washed with distilled water and magnetically separated for several times to remove the excess of CA.

In the third step, the CA-modified NPs were coated with silica, using tetraethyl orthosilicate (TEOS) (Campelj et al (2009)). 10 mL of CA-modified NPs aqueous solution were mixed in a $20 \mathrm{~mL}$ ethanol solution (35\% v/v) containing 3\% (v/v) TEOS. The $\mathrm{pH}$ value of the solution was maintained at 11 for $90 \mathrm{~min}$.

\section{Characterization Methods}

A LEO 906E electron microscope operating at $100 \mathrm{KeV}$, was used for transmission electron microscopy characterization. The samples were prepared by deposition of a droplet of particles solution on a copper grid coated with carbon and allowed to dry. Dynamic light scattering (DLS) and $\zeta$ potential were performed with a Zetasizer Nano ZS (Malvern instruments), provided by a $\mathrm{He} / \mathrm{Ne}$ laser of $633 \mathrm{~nm}$ wavelength. The NPs dispersion was analyzed in a polystyrene cell and in a folded capillary cell, for size distribution and zeta potential measurements, respectively. The $\zeta$ potential values were calculated using the Smoluchowski equation (Delgado et al. 2005). The NPs water dried sample was characterized by X-ray diffraction with a Bruker D8 Discover diffractometer using $\mathrm{Cu} \mathrm{K \alpha}$ incident radiation. Hysteresis loops of the obtained nanoclusters at room temperature were measured with a superconducting quantum interference device (SQUID) magnetometer (Quantum Design MPMS5XL). The experimental results were corrected subtracting the sample holder's diamagnetic contribution.

\section{Magnetophoresis Experiments}


The magnetophoresis setups employed in our experiments are the SEPMAG LAB 1x25 ml 2042 and 2042 Plus systems (Sepmag Technologies). The system consists on a cylindrical cavity containing a high permanent magnetic field with a uniform horizontal gradient pointing toward the walls of the cylindrical vessel.

. The magnetophoresis experiments are performed at Low Gradient Magnetic Field (LGMF) $(<100 \mathrm{~T} / \mathrm{m})$ by placing a bottle of radius $1.5 \mathrm{~cm}$ containing $25 \mathrm{ml}$ of NPs aqueous solution inside the SEPMAG cylindrical cavity. The initial yellow-brown dispersion becomes transparent progressively, reaching a transparent final state with all particles close to the walls of the bottle. Opacity measurements were performed using the external light source SEPMAG CBL Q250 ml (Sepmag Technologies). Typical magnetophoresis curves consist on a plateau corresponding to the reversible aggregation time followed by a progressive decay of the suspension opacity until 100\% transparency is reached (De la Cuevas et al. 2008).

\section{Results and discussion}

Figures $1 \mathrm{~A}$ and $1 \mathrm{~B}$ show the transmission electron microscopy images of the $\mathrm{Fe}_{2} \mathrm{O}_{3}$ silica coated nanoclusters. The aggregation of the nanoclusters occurs during the drying process for TEM imaging. Figure 1C shows the diffractogram of the as synthesized NPs. Because of the modest amount of the sample available, the peaks of the diffractogram are not very well defined. They can be indexed either to maghemite $\left(\gamma-\mathrm{Fe}_{2} \mathrm{O}_{3}\right)$ or magnetite $\left(\mathrm{Fe}_{3} \mathrm{O}_{4}\right)$. In order to simplify the nomenclature, and considering the high probability of the oxidation of the $\mathrm{Fe}_{3} \mathrm{O}_{4}$ into $\gamma$ - $\mathrm{Fe}_{2} \mathrm{O}_{3}$ (Laurent et al. 2008), the nomenclature was fixed on $\mathrm{Fe}_{2} \mathrm{O}_{3}$, instead of $\mathrm{Fe}_{3} \mathrm{O}_{4}$ and $\gamma-\mathrm{Fe}_{2} \mathrm{O}_{3}$. 

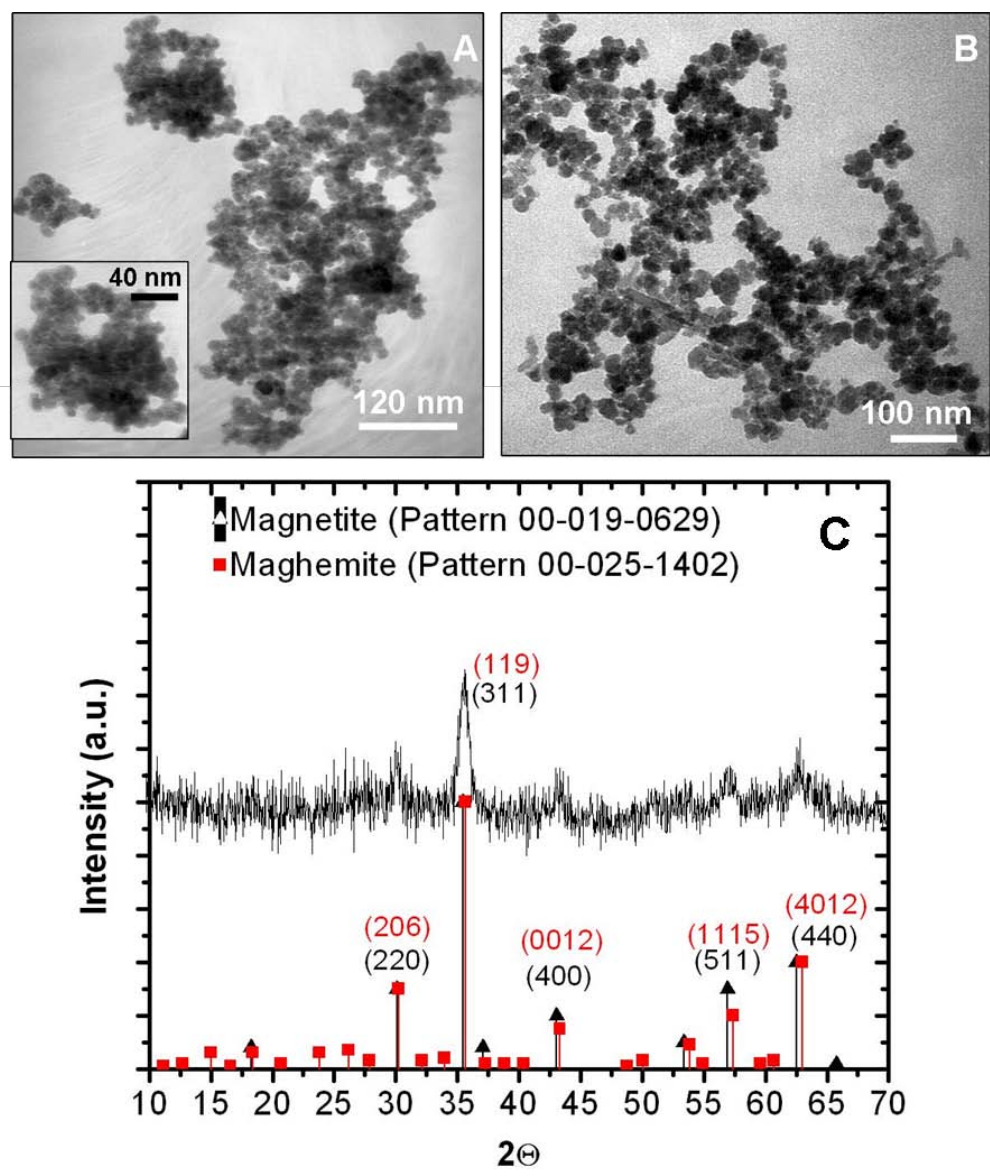

Fig. 1 Typical TEM images of silica coated nanoclusters (A, B). The aggregation of the nanoclusters occurs during the drying process for TEM imaging. XRD pattern of the as synthesized NPs (C).

DLS measurements indicate an average size cluster distribution of $150 \mathrm{~nm}$. Magnetic measurements on a silica coated nanoclusters sample are shown in Figure 2. The regime of saturation starts at about $1 \mathrm{KOe}(0.1 \mathrm{~T})$ with a saturation magnetization value Ms of $44 \mathrm{emu} / \mathrm{g}$. Due to the magnetic field gradient values used in this study, $15 \mathrm{~T} / \mathrm{m}, 30 \mathrm{~T} / \mathrm{m}$ and $60 \mathrm{~T} / \mathrm{m}$, the absolute values of the magnetic field within the experimental systems varies radially from 0 to $0.225 \mathrm{~T}$, 0 to $0.45 \mathrm{~T}$, and 0 to $0.9 \mathrm{~T}$ respectively, being zero at the centre and maximal at the cylindrical walls. At the instant zero (when the bottle is inserted on the magnetophoresis device) only the NPs nearer the axis are not saturated. Once the process starts, the NPs move radially and in few seconds all the NPs are in the region where the magnetic field is high enough to saturate them. The amount of the volume with 
non saturated NPs at the starting time is below $20 \%$ for the $15 \mathrm{~T} / \mathrm{m}$ system, less than $5 \%$ for the $30 \mathrm{~T} / \mathrm{m}$ and less than $2 \%$ for the $60 \mathrm{~T} / \mathrm{m}$

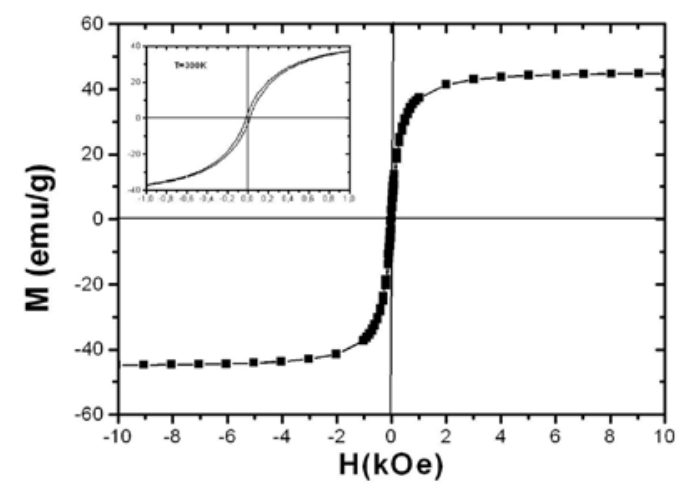

2 Magnetization curve of $\mathrm{Fe}_{2} \mathrm{O}_{3}$ silica coated nanoclusters at room temperature

\section{Magnetophoresis behaviour}

Figure 3 shows the magnetophoresis curves of the obtained nanoclusters at different magnetic gradients: $15 \mathrm{~T} / \mathrm{m}, 30 \mathrm{~T} / \mathrm{m}$, and $60 \mathrm{~T} / \mathrm{m}$. The total separation time depends on the magnetic force applied inside the SEPMAG system. For the $15 \mathrm{~T} / \mathrm{m}$ gradient the aggregation time takes about 100s. This time decreases one order of magnitude ( 10s) when a $60 \mathrm{~T} / \mathrm{m}$ gradient is applied. No relevant differences between the aggregation times corresponding to $60 \mathrm{~T} / \mathrm{m}$ and $30 \mathrm{~T} / \mathrm{m}$ applied magnetic gradients are observed. The magnetophoresis separation is induced by reversible aggregation of the nanoclusters. This reversible aggregation strongly depends on the total interaction potential between the particles, magnetization and size of the nanoclusters, as well as on the concentration of the suspension (De la Cuevas et al. 2008; Faraudo et al. 2010). 

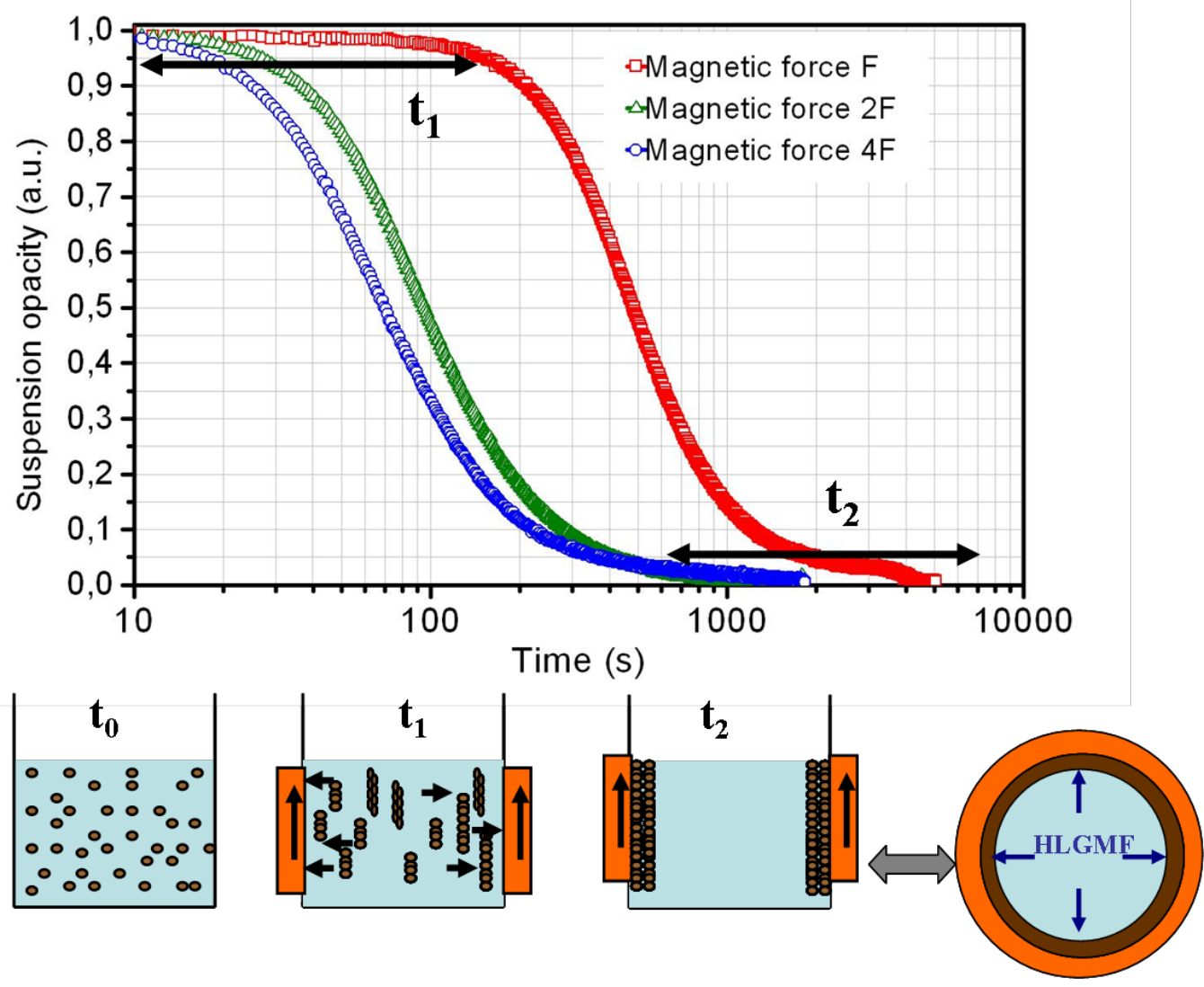

Fig. 3 Magnetophoresis curves of the synthesised $\mathrm{Fe}_{2} \mathrm{O}_{3}$ silica coated nanoclusters at different magnetic field gradients: $15 \mathrm{~T} / \mathrm{m}, 30 \mathrm{~T} / \mathrm{m}$ and $60 \mathrm{~T} / \mathrm{m}$, corresponding to $\mathrm{F}, 2 \mathrm{~F}$ and $4 \mathrm{~F}$ respectively. At $t_{0}$, before introducing the sample into the separation system, the suspension is homogeneous. At $t_{1}$, aggregates are formed under HLGMF, and start moving to the walls of the bottle. At $t_{2}$, all the nanoclusters are closed to the walls of the bottle. And the buffer could be removed to proceed to the following step of the process if suitable

By applying $60 \mathrm{~T} / \mathrm{m}$, at $\mathrm{pH} \sim 10.5$, the aggregation time increases from less than 1s for as synthesized NPs to 300s for CA-modified NPs (Fig. 4). The $\zeta$ potential of the as synthesized NPs and the CA-modified NPs is $-20 m V$ and $-43 m V$, respectively. These results suggest the strong influence of the electrostatic stabilization in the magnetophoresis process. High electrostatic stabilization guarantees a more stable suspension, but induces a larger separation time. 


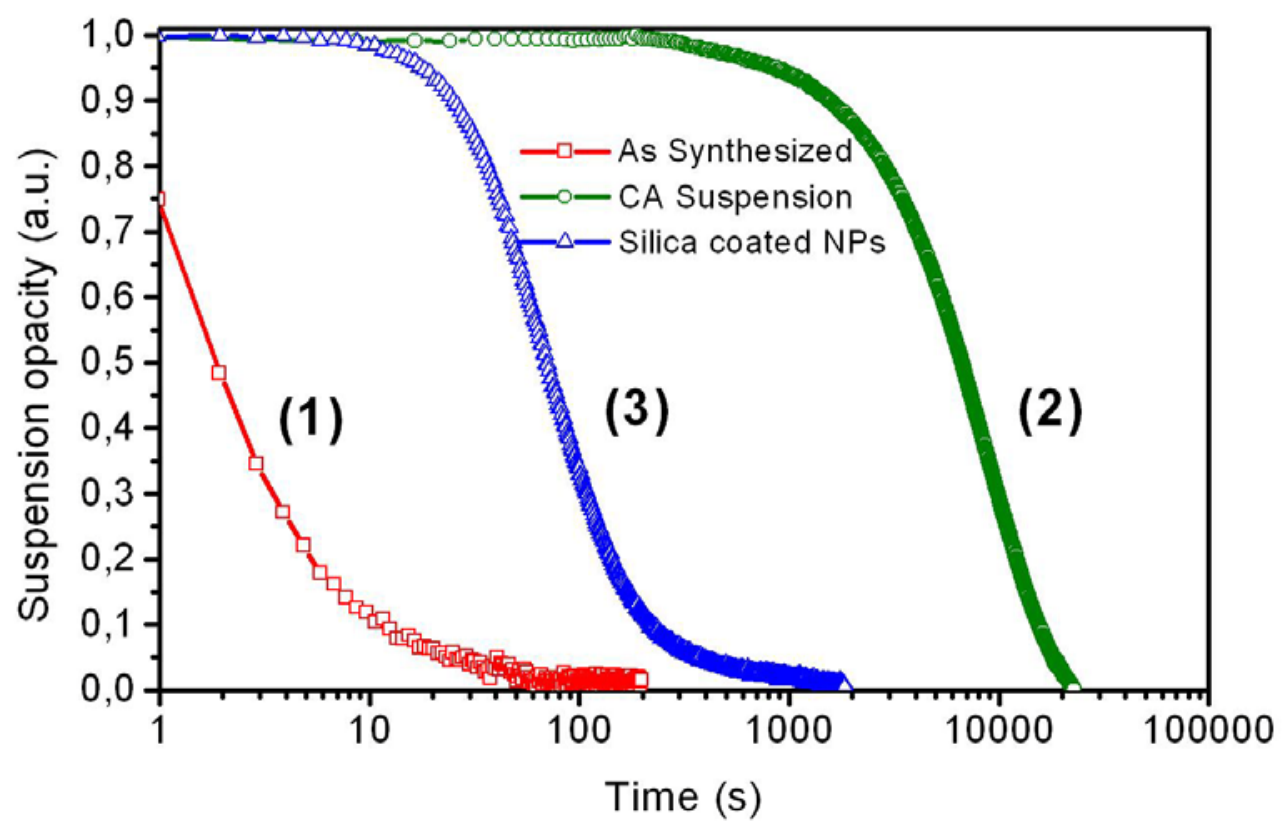

Fig. 4 Reversible aggregation times of NPs at each step of the synthesis route: as synthesized NPs (1), CA-modified NPs (2) and the obtained $\mathrm{Fe}_{2} \mathrm{O}_{3}$ silica coated nanoclusters (3).

In the case of the $\mathrm{Fe}_{2} \mathrm{O}_{3}$ silica coated nanoclusters, the aggregation time takes 10s (Fig. 4) and the $\zeta$ potential is $-30 \mathrm{mV}$. The theoretical limit of stability in a colloidal system is $|30| \mathrm{mV}$. Colloidal systems show good stability if its $\zeta$ potential is higher than $30 \mathrm{mV}$ or lower than $-30 \mathrm{mV}$ (Taboada et al. 2007). This result indicates that the synthesized nanoclusters in the present work are at the limit of stability in colloidal systems.

\section{Effect of $\mathrm{pH}$ on the Reversible Aggregation Process}

As shown in Fig. 5, CA has three dissociable protons associated with its three carboxylate groups. It has been claimed that CA acts as an adsorbate tribasic acid on metal oxides (adsorbent) (Kallay et al. 1985). The deprotonation of CA depends on the $\mathrm{pH}$ of the suspension. At $\mathrm{pH} 5.5$, CA appears mainly with two deprotonated species $\left(\mathrm{AH}^{2-}\right)$ and then is adsorbed onto the NPs surface positively 
charged (Taboada et al. 2007). At pH 10, CA appears at their fully deprotonated state $\left(\mathrm{A}^{3-}\right)$. As reported by Hadju et al (2009), the CA may be adsorbed on the surface of the NPs by coordinating via one or two of the carboxylate functionalities depending on the steric necessity and the curvature of the surface. When the CA affinity limit is reached, the CA adsorption increases further and a rearrangement of CA binding towards a tighter packed surface structure can occur. Only one carboxylate group of CA will be bound to the NPs surface. In these conditions, there will be at least one carboxylic acid group exposed to the solvent that should be responsible for the surface charge. Furthermore, the presence of a terminal carboxylic group provides a possible way of extending the bond formation (Goodarzi et al. 2004). This chemisorption process provides the electrostatic stability of the suspension.

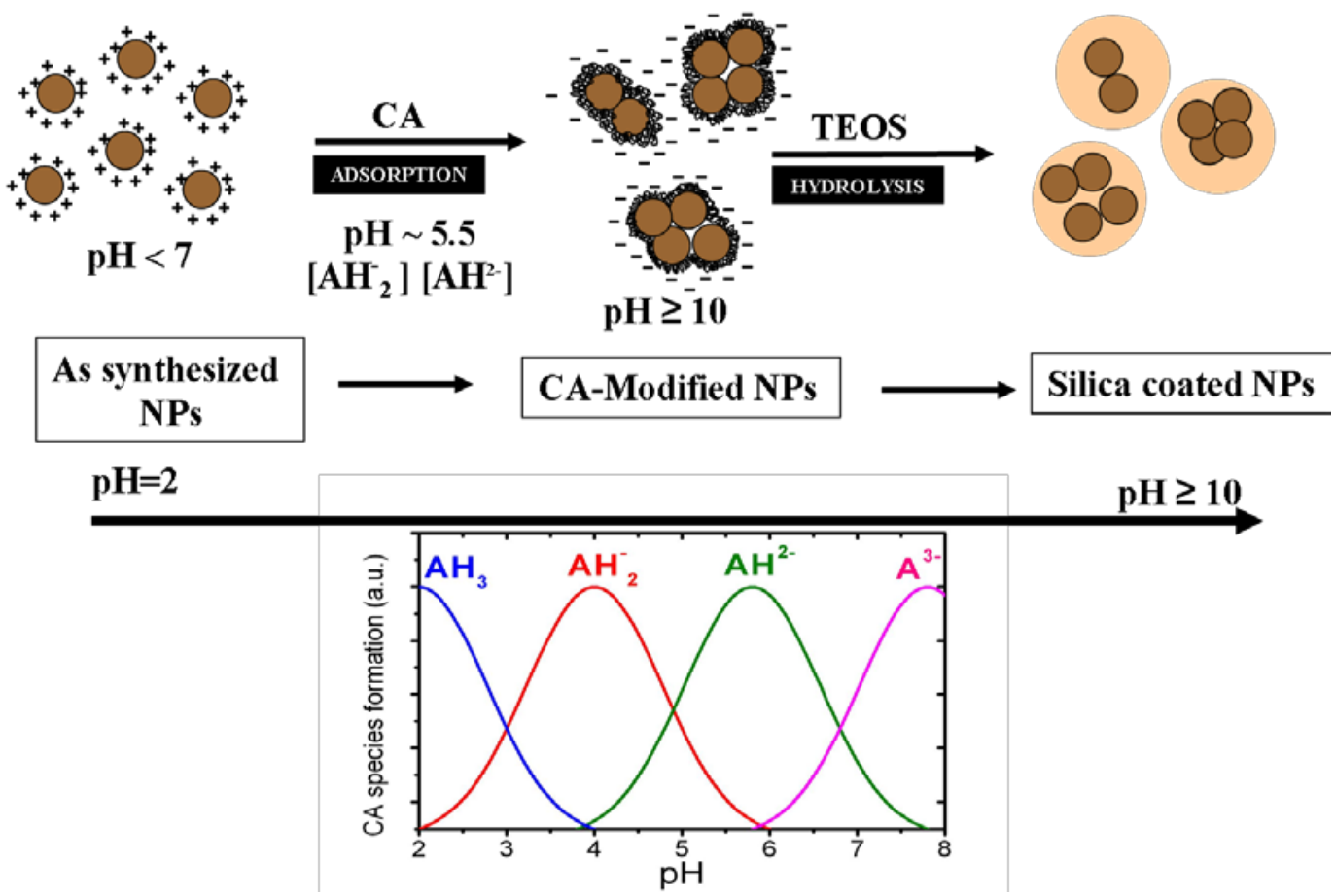

Fig. 5 Schematic representation for the synthesis of NPs, using CA to produce aqueous stable suspension

Different samples of CA-modified NPs with different $\mathrm{pH}$ in the range 2-10 were prepared separately. Samples with $\mathrm{pH}<3$ were not considered for this study as 
NPs degradation and cluster formation are observed. This behaviour could be attributed to the suppressed dissociation of CA carboxylic groups in this acidic region. The DLS measurements show homogeneous aggregates of the CAmodified NPs of about $40-45 \mathrm{~nm}$. These aggregates could be induced by the chemisorption process of CA onto NPs (Kallay et al. 1985; Hadju et al. 2009). The aggregates size is not significantly affected by $\mathrm{pH}$ changes in the considered range.

A similar study was developed in the case of the non modified NPs. The working pHs were 9, 7.17 and 4.78. In this case, the DLS measurements showed that the size of the aggregates is about $65 \mathrm{~nm}$.

On the other hand, according to Fig. 6, charge distribution of both the as synthesized NPs and CA-modified NPs strongly depends on the $\mathrm{pH}$ of the suspension. Fig. 6 (right) shows that acidic pHs induces considerable changes in the CA-modified NPs due to the suppressed dissociation of CA carboxylic groups, which are responsible for the changes of NPs surface. At pHs 6.75 and 4.62 a new peak appears in the $\zeta$ potential curves at about $-10 \mathrm{mV}$ indicating that the suspension is becoming electrostatically heterogeneous. At basic $\mathrm{pH}$ (8.43), the $\zeta$ potential curve shows a narrow peak at about $-43 \mathrm{mV}$ indicating the stability of the suspension. 

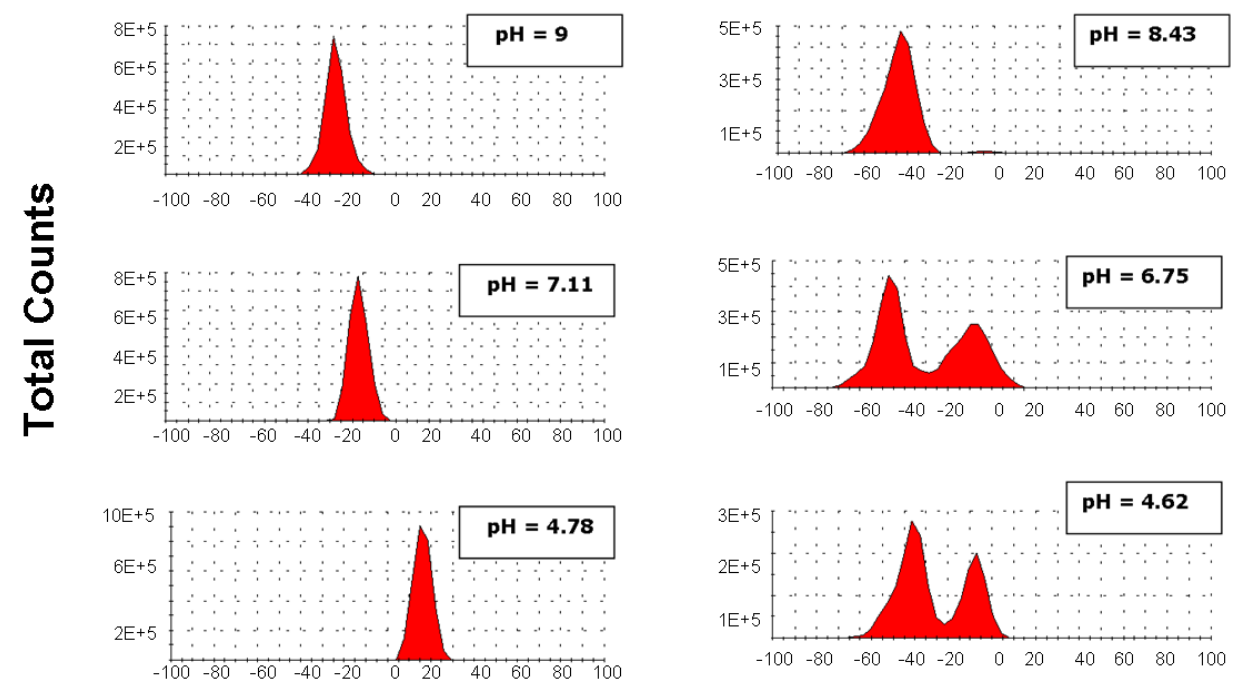

Z Potential (mV)

Fig. $6 \zeta$ potential measurement at different $\mathrm{pH}$ values of the as synthesized NPs (left) and the CA-modified NPs (right)

In the case of as synthesized NPs, $\zeta$ potential is sensitive to the $\mathrm{pH}$ values of the suspensions $(16.9 \mathrm{mV}$ at $\mathrm{pH}=4.78,-17 \mathrm{mV}$ at $\mathrm{pH}=7.17$ and $-23,37 \mathrm{mV}$ at $\mathrm{pH}=9)$. However, no split-peak of the $\zeta$ potential curves was observed.

As previously mentioned, a typical magnetophoresis curve of CA-modified NPs at basic $\mathrm{pH}$ presents a unique plateau corresponding to the reversible aggregation time, followed by a progressive decrease of the suspension opacity. As shown in Fig. 7, at pHs 6.72 and 4.62, the magnetophoresis curves show a first plateau followed by a progressive decay of the suspension opacity until $\sim 50 \%$ and a second plateau followed by a progressive decay of the opacity until $100 \%$ of the suspension transparency is reached. These results are in agreement with the $\zeta$ potential measurement and suggest that the separation process occurs in two steps by the separation of a first group of NPs with a lower $\zeta$ potential followed by the separation of the NPs with higher $\zeta$ potential. At lower $\zeta$ potential, the attractive magnetic interactions overcome the electrostatic repulsion and the time separation is reduced. 


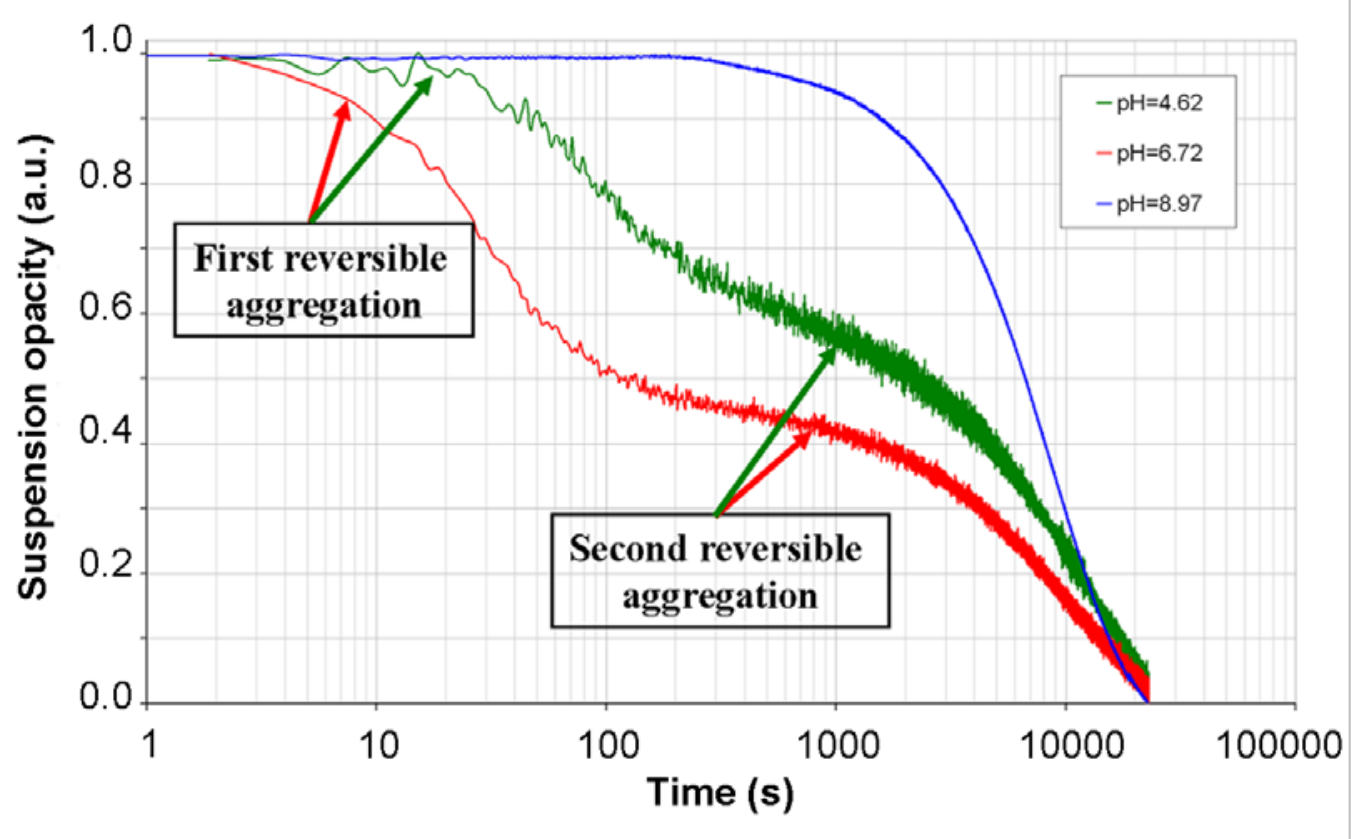

Fig. 7 Magnetophoresis behaviour at 60T/m of CA-modified NPs at different $\mathrm{pHs}$ of the suspension. The curves show a first plateau corresponding to the aggregation time of the particles with lower $\zeta$ potential and a second plateau corresponding to the aggregation process of the NPs with higher $\zeta$ potential

In the case of the as synthesized NPs, as shown in fig. 3, the full magnetophoresis process takes less than 10s, making difficult a comparative monitoring of the aggregation times at the different pHs. These results demonstrate that the destabilization and deterioration of the magnetic suspension due to the changes of electrostatic charges on the surface of NPs could be monitored during magnetophoresis process.

\section{Conclusions}

This paper reports on the effects of the stabilizing electrostatic energy on the magnetophoresis process at HLGMF $(<100 \mathrm{~T} / \mathrm{m})$. The experiments demonstrate that $\zeta$ potential strongly affects the separation time of the NPs in several orders of magnitude. Total separation time shifts from $\sim 1.5 \mathrm{~min}$ for the as-synthesized NPs 
$(-20 \mathrm{mV})$ to $\sim 330 \mathrm{~min}$ for CA-modified NPs $(-43 \mathrm{mV})$. Small $\zeta$ potential allows the attractive magnetic interaction to overcome the electrostatic repulsion. This behaviour induces the formation of bigger clusters and the separation time is accelerated.

Modification of the $\zeta$ potential of NPs could be achieved inducing $\mathrm{pH}$ changes of the magnetic suspension. Destabilization and deterioration of the magnetic suspension due to changes of the electrostatic charges on the surface of NPs could be monitored during magnetophoresis process.

\section{Acknowledgments}

The authors thank the Portuguese Foundation of science and technology (FCT) for financial support under grants: NANO/NMed-SD/0156/2007 and PTCD/CTM/69316/2006.

\section{References}

Benjamin CHU (1999) Controlled size polymeric microspheres with superparamagnetic Core International patent WO 99/12000; PCT/US98/21266

Campelj S, Makovec D, Drofenik M (2008) Preparation and properties of water-based magnetic fluids. J Phys: Condens Matter 20204101.

Campelj S, Makovec D, Drofenik M (2009) Functionalization of magnetic nanoparticles with 3-aminopropyl silane. J Mag Mag Mater 321 1346-1350.Chen H, Kaminski, MD, Ebner AD, Ritter JA, Rosengard AJ (2007) Theoretical análisis of a simple yet efficient portable magnetic separator design for separation of magnetic nano/micro carriers from human blood flow J Mag Mag Mater 313 127-134.

De Las Cuevas G, Faraudo J, Camacho J, (2008) Low-gradient magnetophoresis throught field induced reversible aggregation J Phys Chem C 112 945-950.

Delgado A V, Gonzalez-Caballero F, Hunter R J, Koopal L K, Lyklema J, (2005) Measurement and Interpretation of Electrokinetic Phenomena IUPAC technical Report. Pure Appl Chem 77 1753-1805.

Earhart C M, Wilson RJ, White R L, Pormand N, Wang SX (2009) Microfabrication magnetic sifter for highthroughput and high-gradient magnetic separation. J Mag Mag Mater 321 1436-1439

Faraudo J, Camacho J (2010) Cooperative magnetophoresis of superparamagnetic colloids: theoretical aspects. Colloid Polym Sci 288 207-215.

Friedman G, Yellen B B (2005) Magnetic separation, manipulation and assembly of solid phase in fluids. Current Opinion in Colloid \& Interface Science 10 158-166.

Goodarzi A, Sahoo Y, Swihart MT, Prasad PN (2004) Aqueous Ferrofluid of Citric Acid Coated Magnetite Particles. In proceedings of Mat Res Soc Symp 789 6.6.1-6.6.6. 
Hadju A, Illes E, Tombacz E, Borbath I (2009) surface charging, polyanionic coating and colloid stability of magnetite nanoparticles. Colloids \& surf A: Physicochem. Eng. Aspects 347 104-108

Kallay N, Matijevic E (1985) Adsorption at solid/solution interfaces. 1. interpretation of surface complexation of oxalic and citric acids with Hematite. Langmuir 1 195-201.

Laurent S, Forge D, Port M, Roch A, Robic C, Elst L V, Muller R N (2008) Magnetic Iron Oxide nanoparticles: Synthesis, Stabilization, Vectorization, Physicochemical characterization, and biological applications. Chem Rev 108 2064-2110.

Sayed ZM, Telang SD, Ramchand CN (2003) Application of magnetic techniques in the field of drug delivery and biomedicine. BioMagnetic research and Technology 1:2.

Sepmag tecnologies www.sepmag.eu

Siraprapa M, Gamolwan T, Uthai W, Metha R (2008) Magnetite nanoparticles stabilized with polymeric bilayer of poly(ethylene glycol) methyl ethter-poly(ع-caprolactone) copolymers Polymer 49 3950-3956.

Smistrup K, Lund-Olesen T, Hansen M.F, Tang PT (2006) Microfluidic magnetic separator using an array of soft magnetic elements J Appl Phys 99 08P102.

Taboada E, Rodriguez E, Roig A, Oro J, Roch A, Muller RN (2007) Relaxometric and magnetic characterization of ultrasmall iron oxide nanoparticles wth high magnetization. Lagmuir 23 4583-4588.

Tartaj P, Del Puerto M M, Veintemillas-Verdaguer S, Gonzalez-Carreno T, Serna C J (2003) The preparation of magnetic nanoparticles for applications in biomedicine. J Phys D: Applied physics 36 R182-R197.

Wilson K S, Goff J D, Riffle J S, Harris LA, St Pierre T G (2005) Polydimethylsiloxane-magnetite nanoparticle complexes and dispersions in polysiloxane carrier fluids Polym Adv Technol 16 200-211.

Wilson KS, Harris LA, Goff JD, Riffle JS, Dailey JP (2002) A generalized method for magnetite nanoparticle steric stabilization utilizing block copolymers containing carboxylic acids. European Cells and Materials 3 suppl 2 206-209. 